\title{
Non-wet kingfisher flying in the rain: The tumble of droplets on moving oriented anisotropic superhydrophobic substrates
}

Yihua Zhenga, Chengchun Zhanga, b, *, Jing Wang ${ }^{\mathrm{c}}$, Liang Yang ${ }^{\mathrm{d}}$, Chun Shen ${ }^{\mathrm{a}}$, Zhiwu Han ${ }^{\mathrm{a}}$, Yan Liu ${ }^{\mathrm{a}}$

${ }^{a}$ Key Laboratory of Bionic Engineering (Ministry of Education), Jilin University, Changchun 130022, China

${ }^{\mathrm{b}}$ State Key Laboratory of Automotive Simulation and Control, Jilin University, Changchun,130022, China

${ }^{c}$ College of Physics, University of Leeds, Changchun 130012, China

${ }^{d}$ Centre for Renewable Energy Systems, Cranfield University, Cranfield MK43 OAL, United Kingdom

\section{ABSTRACT}

Extensive studies of anti-wetting have been restricted to stationary substrates, while dewetting mechanisms on moving interfaces are still poorly understood. Due to the hydrophobic and anisotropic surface characteristics of kingfishers, they are able to easily change flight direction even under highintensity precipitation. The present study aims to mechanistically analyze how the synergy of interfacial movement, anisotropy, and superhydrophobicity affects rapid dehydration. We have designed a droplet-conveyor system to simulate the bouncing of droplets on moving anisotropic superhydrophobic targets and performed simulations via the lattice Boltzmann algorithm. The moving interface can induce a directional tumbling behavior of the droplet and effectively avoid continuous wetting in the same region. We found that droplet tumbling is essentially caused by transformed depinning velocity vectors at interface the downstream. Also, the hang time of a tumbling droplet is positively related to the angle between the motion vector and the texture. The oriented anisotropic motion facilitates the tumbling of droplets and decreases their hang time by up to $23 \%$ as compared to that on a stationary inclined superhydrophobic surface. Similar interfacial process dehydration also occurs on a non-wet kingfisher flying in the rain, and we believe that these findings provide valuable new insights for high-efficiency water repellency of surfaces.

KEYWORDS: droplet-conveyer, rapid-dewetting, superhydrophobic anisotropy, de-pinning, kingfisher

\section{INTRODUCTION}

Raindrops hitting the wings of birds can severely disrupt flight balance by creating additional momentum and torque ${ }^{1}$, particularly for lightweight birds with low rotational inertia. The occurrence of adherent water caused by rainfall may significantly increase aerodynamic drag. Previous studies of bats have shown that flight energy costs dramatically increase after a heavy rain ${ }^{2}$. Experiments have also shown that wetted feathers increase wing loading during takeoff; thus, sea birds tend to keep their feathers dry ${ }^{3}$. However, some small volant taxa, such as Wilson's storm petrel (Oceanites oceanicus), 
can maintain high-speed and low-drag flight in severe storms ${ }^{4}$. Moreover, the excellent hovering performance of hummingbirds during rainfall has also been confirmed in a laboratory setting ${ }^{5}$. Kingfishers can dive and then exit the water-air interface to accomplish foraging yet remain dry even during rain (Figure 1, see Supporting Movie 1) ${ }^{6}$. As rainfall appears to create no potential challenges for small taxa, their striking water-repellent mechanism has been a long-term focus for researchers. Much like the lotus leaf effect, which is characterized by a self-cleaning superhydrophobic function ${ }^{7}$, both a high apparent contact angle $\left(\mathrm{CA}=160^{\circ}\right)$ and resistance to water penetration have been observed in the twofold-structure of pigeon feathers ${ }^{8,9}$. Feathers exhibit excellent anisotropic wettability, due to which droplets flow more easily and transfer along the veins ${ }^{10}$, helping to keep birds dry in the rain.

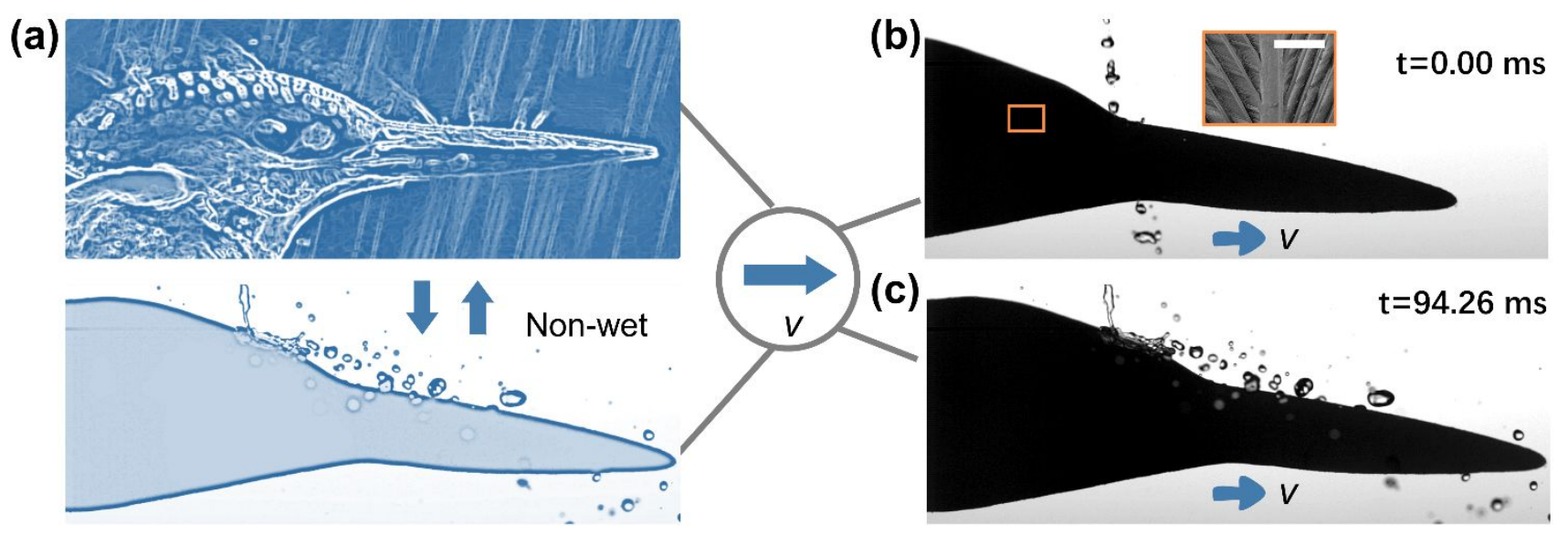

Figure 1. Remarkable water repellency of kingfishers in both natural and laboratory environments. (a) Heavy rain appears to have no effect on a kingfisher, and its feathers remain dry despite the splashing of raindrops in various directions. (b-c) A liquid jet impacting a moving kingfisher under laboratory conditions $(v=0.13 \mathrm{~m} / \mathrm{s})$. The liquid jet is split into droplets of multiple sizes and then bounces off directionally, with no residual moisture being observed on the kingfisher (see Supporting Movie 1). The scale bar represents $400 \mu \mathrm{m}$. Refer to section S3 in the Supporting Information.

The feather dehydration state of birds is a synergistic effect of multiple dynamic factors, of which the static water-repellency of feathers is not the sole feature. Flying Anna's hummingbirds have developed a robust wetting control strategy that regulates the oscillation frequency of the various parts of their body. Combined with the flexibility of their hydrophobic feathers, this mechanism works to break the balance between centrifugal and capillary forces. The elasticity of kingfisher feathers has recently been confirmed to reduce the contact time of the solid-liquid interface by completely modifying the receding dynamics of a droplet during flight ${ }^{11}$. Therefore, the water-repellent mechanism of the coupling between anisotropic hydrophobic feathers and the movement before 
takeoff or during flight may be beneficial to the reduction of any negative effects caused by added moisture mass, such as diminished aerodynamic performance. So far, many studies have only explored the bionic manufacture and wetting of stationary substrates ${ }^{12-21}$, but obviously, the dewetting previously described processes all occur on birds' moving exteriors, as they are movable. Contemporary research of the wetting phenomenon on anisotropic superhydrophobic substrates (ASHSs) has revealed that the novel droplet morphology and significant reduction in solid-liquid contact time are attributed to the macroscopic, anisotropically-textured surface. Yet the dynamic wetting behavior of movable substrates has been analyzed only recently by Almohammadi and Buksh et al. ${ }^{22-24}$, who confirmed that the occurrence of an asymmetric droplet interaction results in a smaller wetted area while a superhydrophobic substrate is being impacted. To the best of our knowledge, no previous works have involved how linear motion vectors affect surface water repellency. Thus, the mechanism of water repellency of moving substrates, especially on anisotropic surfaces, is still far from being understood.

In this study, the influences of various natural factors, such as vibrating winds and differences in species diversity, can be dramatically reduced or even eliminated in a laboratory environment, thereby facilitating the analysis of the water-repellent mechanisms of moving substrates. We prepared anisotropic superhydrophobic copper plates and designed a conveyor belt to drive the substrate to maintain linear motion, in order to simulate the dewetting behavior of the kingfisher flying in the rain. How the droplet bounce dynamics is affected by the angle between the surface texture direction and the motion vector is investigated. Also, the relationship between the asymmetrical "pinning" effect and the direction of the motion vector were given, and we have summarized the mechanism of the tumbling droplets induced by unbalanced de-pinning effects.

\section{EXPERIMENT AND METHODS}

\subsection{Droplet impact configuration and data analysis}

To provide additional details in this experiment, an indoor analysis system consisting of two highspeed cameras was installed in both the vertical and horizontal directions, as shown in Figure $2 \mathbf{a}$. Deionized water (extra interference, e.g., chloride, was eliminated) was used to generate droplets in conjunction with a needle-syringe-stepper motor system controlled by a computer. Using this system, droplets of $2.06 \mathrm{~mm}$ in diameter were adjusted to fall from various heights of $h_{i}=6-46 \mathrm{~mm}(i=1,2, \ldots$, $25, v=0.34-0.95 \mathrm{~m} / \mathrm{s}$ ). The corresponding crucial dimensionless parameters that characterize sensitive hydrodynamic phenomena are listed in Table S2 in the Supporting Information; the selected physical parameters of the water at an indoor temperature included an initial diameter $D_{0}$ of $2.06 \mathrm{~mm}$, a density 
$\rho$ of $997 \mathrm{~kg} / \mathrm{m}^{3}$, a dynamic viscosity coefficient $\mu$ of $0.0009 \mathrm{~Pa} \cdot \mathrm{s}$, and a surface tension $\gamma$ of $0.072 \mathrm{~N} / \mathrm{m}$. The Weber number ( $W e=\rho v^{2} D_{0} \gamma=3.3-25.6$ ) is usually used to characterize the equilibrium relationship between inertial force and surface tension during the droplets impacting experiments. Here, $W e$ is varies only if different height for droplet free fall.

The target substrate was mounted on a conveyor belt with uniform rectilinear motion at $0.13 \mathrm{~m} / \mathrm{s}$. To simulate the relative true state of raindrops impacting a feather, the configurations of the three selected typical substrates included the angle between the direction of the grooves with velocity vectors of $0^{\circ}, 45^{\circ}$, and $90^{\circ}$, respectively, as shown in Figure $\mathbf{2 b}$. In addition, the analysis of droplets impacting a stationary ASHS was also taken into account for comparative verification.

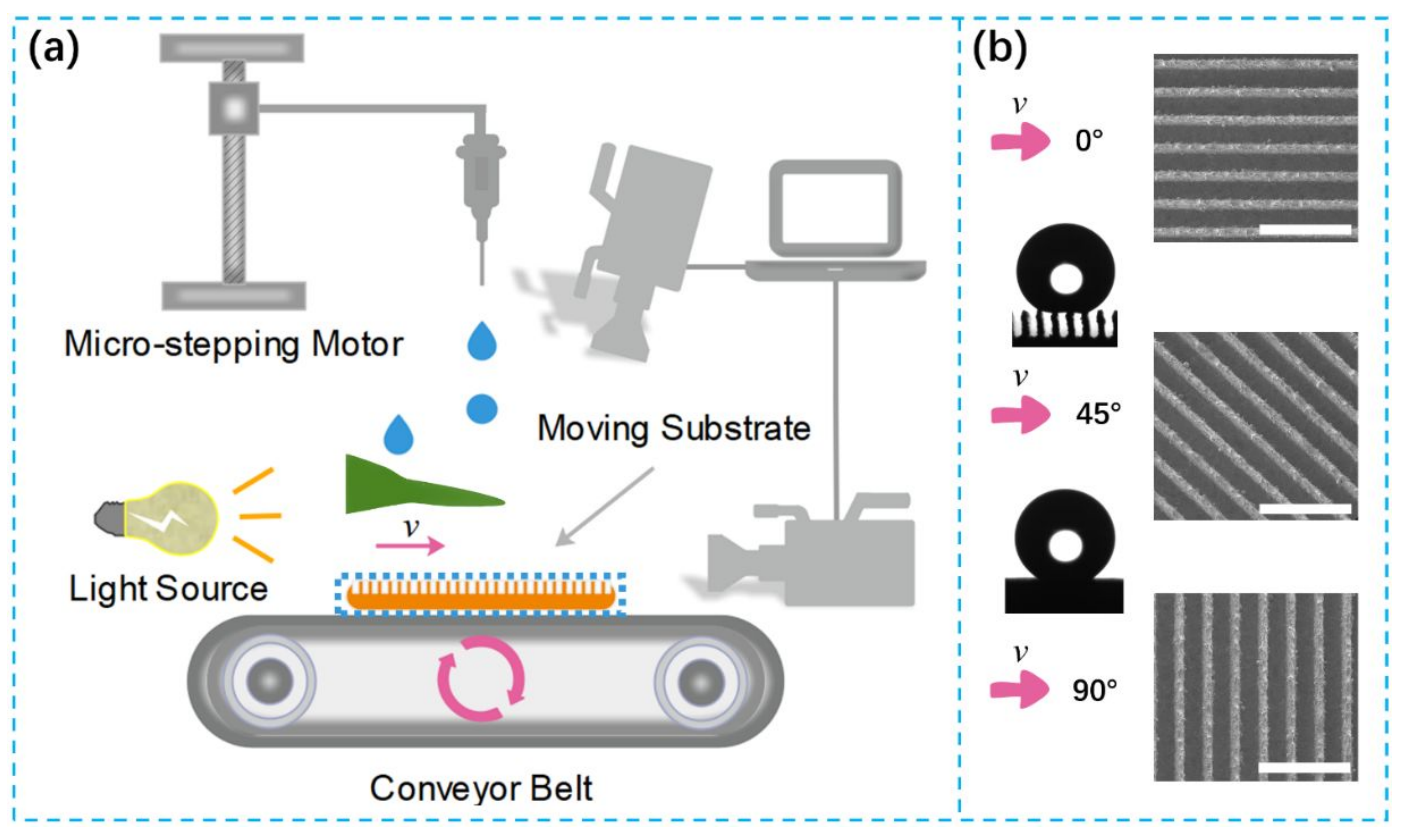

Figure 2. Schematic diagram of droplets impacting the three selected moving anisotropic substrates. (a) Schematic diagram of a droplet impinging on a moving target substrate in which the droplet falls and collides with the passing substrate $\left(v_{s}=0.13 \mathrm{~m} / \mathrm{s}\right)$. (b) Configurations of the moving target substrates. The angles $0^{\circ}, 45^{\circ}$, and $90^{\circ}$ can be defined as the angle between the direction of the arrangement of the grooves and the speed vector. This means that the three typical experiences of raindrops impacting feathers are taken into account under normal circumstances. The insets indicate that the contact angles of the substrate were $155^{\circ}$ (from the perspective of the direction along the veining) and $157^{\circ}$ (from the perspective of the direction perpendicular to the veining), respectively. The scale bar for all SEM frames represents $1000 \mu \mathrm{m}$. See Figure S4 for more information.

As the falling droplets impacted the moving target substrate, the visual data was simultaneously captured by two high-speed cameras (Phantom v711, Vision Research, Inc.) with sample rates of 13,000 frames per second, resolutions of $800 \times 600$ pixels, and exposure times of $76.6 \mu$ s. All captured 
videos were then discretized into images and analyzed on a computer using both MATLAB code and the open-source software ImageJ. No splashing phenomenon was observed to ensure that the volume of droplets was maintained under all testing condition, and all experiments were performed at least three times.

\subsection{Fabrication and characterization of target substrates}

Inspired by the superhydrophobic and anisotropy of the kingfisher bird feathers, we fabricated substrates to mimic the corresponding wettability. The design of the ASHS was based on the characterization of kingfisher feathers on the macro scale (Figure S2 in the Supporting Information). The microstructures were characterized by arrays of trenches and ribbings that were fabricated on copper plates (99.9\% purity, purchased from Southwest of copper Co., Ltd., Kunming, China) based on previous observations of the kingfisher feather structure (refer to section S3 in the Supporting Information). A unidirectional groove-textured surface similar to the parallel ribbings of the kingfisher's feathers was formed using wire electrical discharge machining (EDM). The surface was then descaled in acetone (Analytical Reagent, AR), absolute ethanol (AR), and hydrochloric acid (AR), respectively. After further ultrasonic cleaning with absolute ethanol, the surface was treated with $1 \mathrm{H}, 1 \mathrm{H}, 2 \mathrm{H}, 2 \mathrm{H}$-perfluorodecyl trichlorosilane to reduce(fluoridize) its surface energy via chemical vapor deposition ${ }^{25,26}$. All the chemicals mentioned above were purchased from Aladdin Bio-Chem Technology Co., LTD, Shanghai, China. The aim of this process was to fabricate a surface comparable to that of kingfisher feathers while avoiding the pancake bouncing of droplets, which can significantly reduce the solid-liquid interface contact time $t^{27}$. As such, ribbings with a width of $0.1 \mathrm{~mm}$, height of $0.8 \mathrm{~mm}$, and center-to-center spacing of $0.2 \mathrm{~mm}$ were arranged on a 3-mm thick copper plate. The static wettability of the samples was measured using a contact angle analysis system (OCA 20, DataPhysics, Germany), and the microstructure morphology was characterized via scanning electron microscopy (S-4800, HITACHI Corp., Tokyo, Japan). The contact angles of the target substrates were $155^{\circ}$ (from the perspective of the direction along the veining) and $157^{\circ}$ (from the perspective of the direction perpendicular to the veining), and the corresponding sliding angles were $3^{\circ}$ and $7^{\circ}$, respectively (see the insets of Figure $\mathbf{2 b}$ ).

\subsection{Simulated investigation}

Numerical simulations were performed to analyze the velocity fields and morphological changes of the particles inside the droplets, thereby promoting an understanding of droplet dynamics on the moving substrates. To qualitatively analyze the intrinsic phenomenon of the solid-liquid interaction, an algorithm based on lattice Boltzmann methods was implemented using a D3Q19 grid ${ }^{28-30}$. Refer to 
section S1 in the Supporting Information for simulation details.

\section{RESULTS AND DISCUSSION}

\subsection{Coupling effect of the translational motion and oriented included angle}

To verify the dynamic anisotropy of the target substrate, the interaction of a droplet impacting a stationary ASHS was first investigated. When the droplet impacted the stationary anisotropic surface, the dynamic behavior of the droplet was symmetrical from the side view; however, for the ASHS, the asymmetric wetting state was primarily due to the discontinuous topology (e.g., the obvious directional difference in the edge of the solid) on the moving contact line. This process eventually led to the asymmetry of the droplets in the spreading and receding stages. It should be noted that the effect of anisotropy is negligible because the difference in directionality of the droplets is not significant under low Weber number regimes, such as $W e=3.3$ (see Figure S5A and Supporting Movie 2), a finding that is consistent with previous studies on the morphology of droplets impacting superhydrophobic substrates ${ }^{12,31}$. However, an approximately elliptical morphology of a droplet was also observed, and the droplet was found to be more likely to spread along the groove direction rather than the perpendicular direction from the overhead perspective at $W e=14.4$. This finding corresponds well to droplet behavior on other anisotropic substrates ${ }^{32-35}$, as shown in Figure S5B (see Supporting Movie $3)$.

Birds will adjust various flight attitudes to weaken or even eliminate extra flight energy costs during a storm ${ }^{36}$. Diversiform flight attitudes or situations in which variable winds cause the trajectory of raindrops to no longer be vertical inevitably lead to a change in the angle of interaction between the feather and the raindrops. Therefore, the combination of the velocity vector and motion of hydrophobic feathers may have a positive effect on the rapid dehydration of birds. Available statistics on the growth direction of bird feathers are beyond the scope of the present study. Therefore, only three typical cases, namely velocity vectors of $0^{\circ}, 45^{\circ}$, and $90^{\circ}$ (the included angle between the veining and the motion vector), were selected to investigate the mechanism of this coupling effect on efficient hydrophobicity in an indoor laboratory environment.

The morphological symmetry of the droplets during the spreading and receding phases under the selected Weber number regimes and angles was investigated from an overhead view. It was also experimentally confirmed that the included angle coupled with a moving ASHS can significantly affect the droplet interfacial dynamics. Note that directional spreading was significantly suppressed in the direction of the motion vector during the interaction, as illustrated in the areas respectively indicated by green, yellow, and red lines in Figure 3. Images of the droplets spreading to their maximum size at 
the three angles (from left to right) with the increase of the Weber number (from top to bottom) are provided in Figure 3(a-c). The positions of the centers were revised slightly because the droplets did not bounce from the ribbing where they first interacted with the moving ASHS, and are respectively indicated by vertical dashed lines (red, yellow, and green) in Figure 3. The efficiency of the anisotropy and displacement cooperation was not significant, resulting in a symmetrical dynamic droplet topography at a low impact velocity $(W e=3.3)$, as shown in Figure 3a. However, the modification of the wetting dynamics by movement is reflected in the higher Weber number regime $(W e=25.6)$. It can also be observed that the spreading and receding phases of the lamella were thinner downstream at a given time (see the right side of the dotted lines in Figure $\mathbf{3 b}$ and $\mathbf{3 c}$ ), which corresponds reasonably well with the findings of previous studies ${ }^{22,24}$. No distinguishable morphology of the droplets was observed during the spreading phase within $3.00 \mathrm{~ms}$, as illustrated in Figure 3a, and once the droplets began to spread in the direction of the texture and downstream $(t=3.08 \mathrm{~ms}$, Figure $3 \mathbf{b})$, their centers of gravity began to change transiently with the direction of motion. The non-equilibrium wettability of the droplets thus contributed to the direction of the grooves as the velocity of the substrates remained constant ( $t=3.85 \mathrm{~ms}$, Figure 3c). There were two directions of droplets during diffusion, namely one parallel to the velocity and the other perpendicular to the velocity, and it is clear that the spreading velocity of the three-phase contact line was governed by the anisotropy and velocity vector in both directions. Additionally, the retraction force of the droplets is primarily attributed to the central liquid film (with radius $r$ ), which maintained a low surface energy $\left(E_{r} \approx \pi r \gamma^{2}(1-\cos \theta)\right)$ during the receding phase, and could pull the circumferential rim inward ${ }^{37}$. The decrease in $r$ led to an increase in the volume of the circumferential liquid during the recession, resulting in a high value of $E_{r}$ and retraction force. In other words, the central liquid film was continuously elongated and the droplets then exhibited an unbalanced morphology on the moving ASHSs, resulting in a greater $E_{r}$ and retraction force. as shown in Figure 3d, further numerical simulations verified this inference, and revealed that the velocity distribution of the receding droplets is governed by the included angle between the veining and motion vector. Combined with velocity vector analysis, the analyses of the experiments and simulation support this conclusion well (as indicated by the corresponding green, yellow, and red circles at around $4 \mathrm{~ms}$ ). With the increase of the angle, the velocity vector increased at least fivefold, which remarkably suppressed the retraction of the droplet; this was observed on the right side of the droplet as compared to the liquid film on the other side of the central axis. Thus, as illustrated in Figure 4, the synergy between the large retraction force and droplet volume redistribution governed by substrate motion and the included angle of anisotropy drove the droplets obliquely away from the moving substrate (see Supporting Movie 4). 


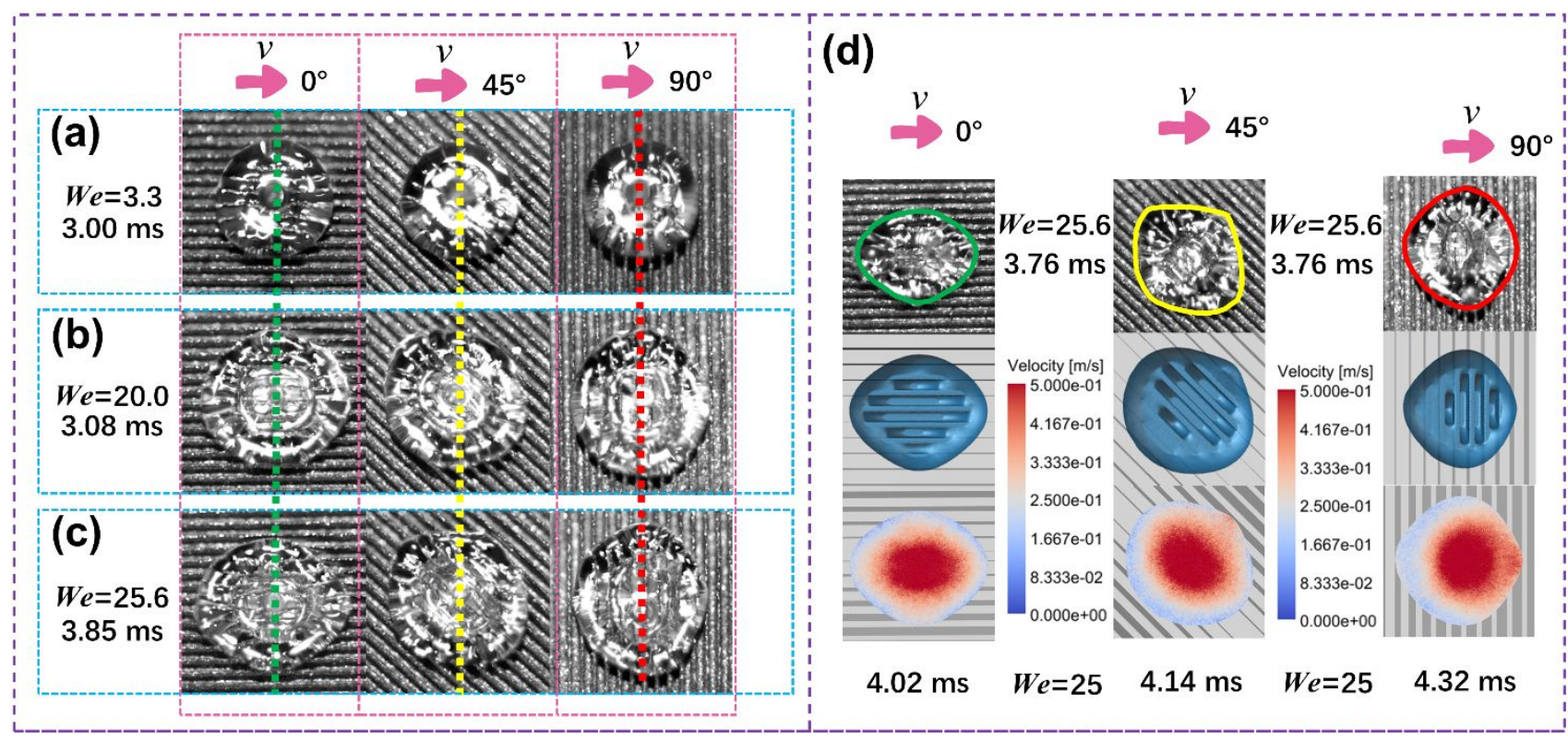

Figure 3. Overhead views of droplets impacting moving substrates with directional anisotropy. (a-c) The coupling of anisotropy and velocity vectors $\left(0^{\circ}, 45^{\circ}\right.$, and $\left.90^{\circ}\right)$ is increasingly noticeable due to the increase in the Weber number (from 3.3 to 25.6). The green, yellow, and red lines are the respective central axes of the droplets, indicating that the morphologies of the droplets are downstream of the substrate displacement. (d) Comparative analysis of the typical coupling effects in experiments and simulations under a high Weber number regime $(\sim 25)$. The simulation analysis confirms that the asymmetric interaction of the droplets is indeed due to the synergy between the velocity and the angle of the texture. Larger inward and upward velocity vectors tend to be distributed downstream in the direction of movement (see Supporting Movie 4).
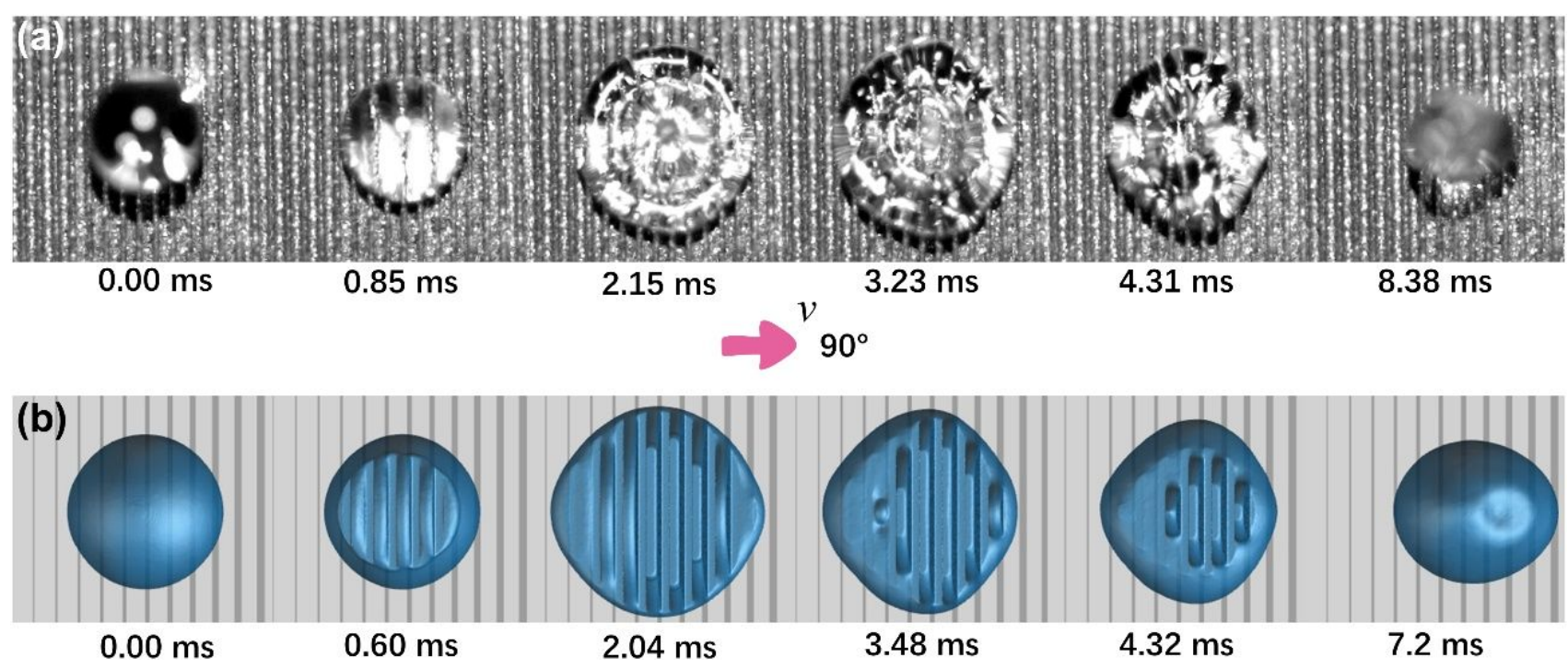

Figure 4. (a) Snapshots of a droplet impacting a moving ASHS ( $W e=20,90^{\circ}, v_{s}=0.13$ ). The significant asymmetric morphology of the droplet occurs after $2.15 \mathrm{~ms}$. (b) The simulation results of 
a droplet with $W e=25$ at a velocity vector of $90^{\circ}\left(v_{s}=0.13\right)$. The non-equilibrium penetration of the liquid into the groove begins at $0.6 \mathrm{~ms}$, and asymmetric permeation adjacent to the substrate ultimately induces an unbalanced morphology of the upper liquid film from a translucent analytical perspective (see Supporting Movie 4).

\subsection{Qualitative and quantitative analysis of enhanced de-pinning effect via the moving ASHS}

It is anecdotally known that the penetration of droplets into a microstructure results in pinning states ${ }^{38}$, which are detrimental to water repellency. A droplet can only penetrate and evacuate the gas between ridges at a higher Weber number $(t=0.62 \mathrm{~ms}$ in Figure S5b), as the downward penetrating force is greater than the stored capillary force, while the stored capillary force is a more dominant power than the upward emptying processes. The trend of the three-phases contact line, in which the more severe the pinning effect, the lesser the ability of the moving contact line to recede, has been used to investigate the extent of the pinning effect ${ }^{22}$.

For the analysis of the penetration and pinning effect of the moving substrates, the circumstance in which the velocity vector is at an angle of $90^{\circ}$ to the direction of the ribbings (corresponding to Figure 2b) was investigated via the optimal side-view visualization. Figure 5a-b show the selected high-speed images of droplets impacting the ASHS with uniform rectilinear motion at $v=0.13 \mathrm{~m} / \mathrm{s}$ when $W e=3.3$ and $W e=14.4$, respectively (see Supporting Movie 5). While the substrate remained moving from left to right (Figure 5a), although the droplet also moved through the spreading, receding, and rebounding process, it exhibited an original differentiation associated with the direction of the velocity vector at $t=2.38 \mathrm{~ms}$ (within the spreading phase). Once the differentiation was initialized, the velocity vector could combine with the substrate to more efficiently modify the moving contact line dynamics. The moving contact line was found to be pinned downstream, whereas it retracted faster upstream, causing the droplet to appear to be inclined downstream during the receding phase (around $4 \mathrm{~ms}$ in Figure 5). A more noteworthy initial asymmetric droplet only occurred as the Weber number regime increased $(W e=14.4)$, and the unbalanced contact line was observed at $t=0.62 \mathrm{~ms}$. A comparison of the diameters of the droplets $D\left(\right.$ scaled as $\left.D_{0}\right)$ at $W e=3.3$ and $W e=14.4$ as a function of the normalized time $t / t_{t h}$ is presented in Figure 6c (see Supporting Movies 2, 3, and 5). The droplets appear to have more easily spread on the movable substrate under both low and high Weber number regimes, and the movement of the contact line stopped at the edge of the solid or encapsulated air as the energy was minimized ${ }^{39}$. Notably, the droplet appears to have been inclined to lift off from the upstream ribbing (marked in red), rather than from the first conflicting ribbing (marked in yellow), which is essentially different from the stationary ASHS. The interval of the two aforementioned ribs 
was then reduced from four to two grooves with the increase of $W e$ to 14.4 (Figure 5b). The penetration of the droplets became more apparent at high Weber numbers, while the penetrative liquid moved with the substrate, and the liquid near the solid edge drove the droplets to the right due to the viscous force. As illustrated in Figure 5 c-d, simulation analysis revealed qualitative similarities from the perspectives of the morphology and the internal particle velocity field. High-speed particles replace the air at the bottom of the groove and collide with the moving solid boundary at $1.54 \mathrm{~ms}$, and the purple and blue rectangles in Figure 5d reveal a typical process of unbalanced de-pinning. The velocity vector of the right portion of the droplet is significantly higher than that of the other side (upstream region) as the droplet is about to lift-off, and it is given the ability to eject to the left. This process indicates that the moving substrate has a greater influence on dewetting than an enlarged wetted area.

(a)

(b)
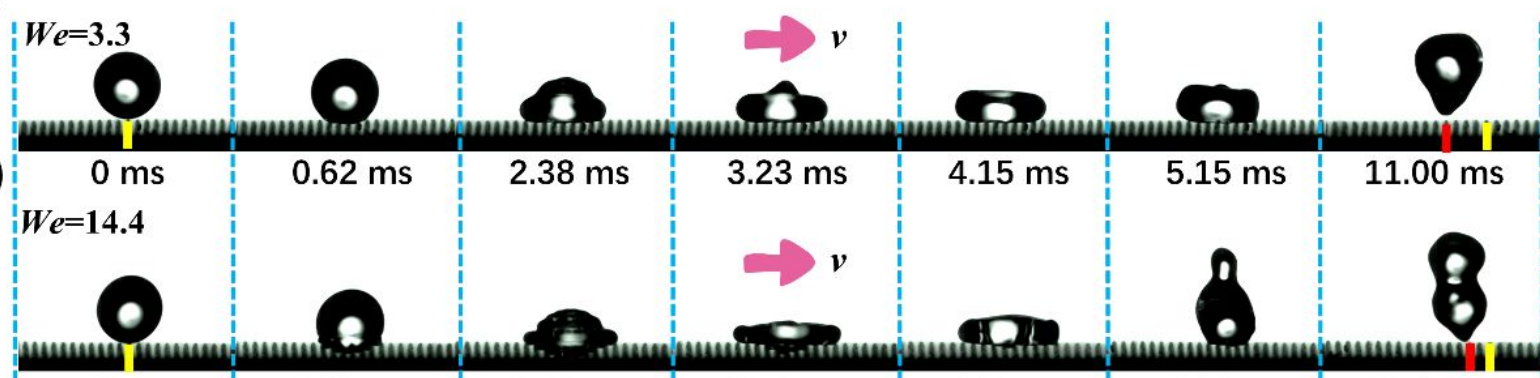

(c)

\begin{tabular}{|c|c|c|c|c|c|c|}
\hline $0 \mathrm{~ms}$ & $0.62 \mathrm{~ms}$ & $1.54 \mathrm{~ms}$ & $2.54 \mathrm{~ms}$ & $4.31 \mathrm{~ms}$ & $7.38 \mathrm{~ms}$ & $11.00 \mathrm{~ms}$ \\
\hline$W e=25.0$ & & & & & \\
\hline & & & & & &
\end{tabular}

(d)

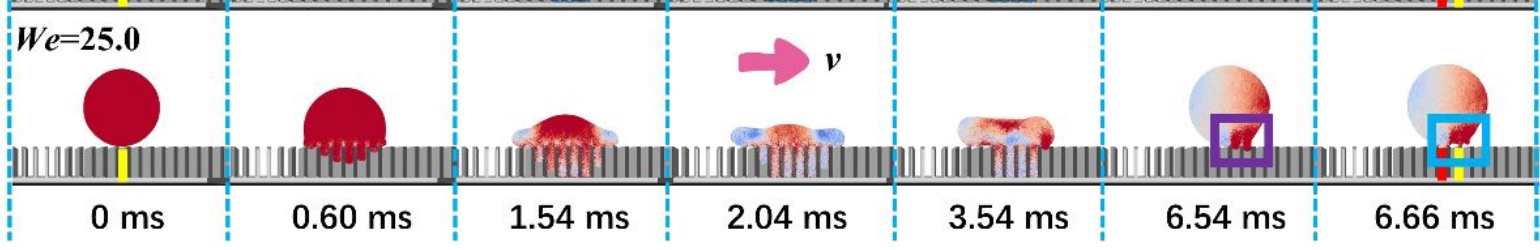

Figure 5. Sequential images of a droplet impacting the moving target ASHS $\left(90^{\circ}\right)$. (a) Side-view images of the impinging of a droplet on the moving ASHS $\left(90^{\circ}\right)$ at $W e=3.3$. The orientation of the droplet was initialized in the direction of the motion vector at $t=2.38 \mathrm{~ms}$, eventually resulting in a slight tilt when receding at $t=5.15 \mathrm{~ms}$. The ribbing site of the initial liquid-solid collision is marked in yellow, while that at which the droplets bounced is marked in red, and the interval between them is four grooves at $t=11.0 \mathrm{~ms}$. (b) Image sequences of a droplet impacting the moving ASHS $\left(90^{\circ}\right)$ at $W e$ =14.4. As the substrate moved to the right, a more severe uneven distribution of mass in the direction of motion occurred at $t=5.23 \mathrm{~ms}$ as compared to that under the low Weber number regimes. Eventually, the interval between the red and yellow lines reduced from four to two grooves at $t=11.0 \mathrm{~ms}$, as compared to that at $W e=3.3$ (see Supporting Movie 5). (c-d) Simulation of droplet interaction and the 
corresponding internal velocity field at $W e=25.0$. The qualitative similarity confirms that a moving ASHS combined with the unbalanced de-pinning effect can both efficiently enhance de-pinning and drive the bouncing droplets upstream. Additionally, wetting does not always occur in the same region, which may reduce the chance of certain areas being wetted (see Supporting Movie 4).

Generally, transverse effective slip lengths can be used to quantitatively verify and estimate the influences of the pinning effect and wettability during liquid-solid interactions ${ }^{40}$. The pinning effect can directionally drive the liquid near the solid wall, resulting in an elongated three-phase contact line. For the non-wetting Cassie state, the anisotropic transverse effective slip length (corresponding to $90^{\circ}$, Figure 2b) can be estimated according to the following equation:

$$
b_{e f f}^{90^{\circ}}=\frac{L}{2 \pi} \frac{\ln \left[\sec \left(\frac{\pi \varphi}{2}\right)\right]}{1+\frac{L}{2 \pi b} \ln \left[\sec \left(\frac{\pi \varphi}{2}\right)+\tan \left(\frac{\pi \varphi}{2}\right)\right]},
$$

where $\varphi$ denotes an evaluation value of the proportions of liquids and gases at the interface, and $b \approx$ $50 \times H$ can be defined as the finite slip length at the interfacial condition (yield gas cushion model). Here $H$ can be estimated as height of macrostructures (the thickness of the gas layer) ${ }^{41}$. However, the slipping at nano-structures $(1 \mathrm{H}, 1 \mathrm{H}, 2 \mathrm{H}, 2 \mathrm{H}$-perfluorodecyl trichlorosilane) is negligible as compared to the magnitude of that at the macro-groove. Combining $\varphi$ and width $W=200 \mu \mathrm{m}$ via image analysis in MATLAB results in the slip transverse effective lengths of $29.4 \mu \mathrm{m}$ and $39.8 \mu \mathrm{m}$ on the stationary substrate at $W e=3$ and $W e=14$, respectively. For the moving ASHS, the corresponding lengths are $32.4 \mu \mathrm{m}$ and $42.7 \mu \mathrm{m}$, which is qualitatively consistent with the conclusions indicated in Figure 5a-b and with previous work ( $23.6 \mu \mathrm{m}$ and $37.5 \mu \mathrm{m}$, respectively $)^{35}$. When the substrate is in translational motion, the results illustrate that the liquid-solid contact areas will likely be no lower than that on the stationary substrate.

However, increasing the wetting area does not mean that the ASHS water-repelling strategy is underestimated when considering that the substrate is movable. A droplet undergoes a receding state before it bounces off the substrate like an elastic ball, and the process of eliminating the pinning effect is embodied by a decreasing three-phase contact line. This asymmetric bouncing has been illustrated as an efficient way to passively reduce the liquid-solid contact time ${ }^{42}$, whereas, in the present study, the symmetry-breaking of the droplets was reconfigured due to the motion of the moving ASHS, and this modification significantly affected the contact time. The interaction between water droplets and a substrate can be regard as a low-viscosity inertia-capillary-dominated system, which directly affects the contact time $t$ and the theoretical contact time $t_{t h}$ of the superhydrophobic substrate ${ }^{43}$ :

$$
t_{t h}=2.6 \sqrt{\rho D_{0}^{3} /(8 \gamma)} \text {. }
$$


This process is not governed by the microstructure of the substrate, and only depends on the initial physical parameters of the droplet, which are $D_{0}=2.06 \mathrm{~mm}$ and $t_{t h}=10.12 \mathrm{~ms}$. It is intuitive to investigate the decline in the number of wetted ribbings from a side view. Figure 6a presents a comparison of the elapsed time (scaled by $t_{t h}=10.12 \mathrm{~ms}$ ) of the de-pinning process, in which the number of wetted ribbings is reduced from three to two. Note that the time spent on de-pinning is consistent with the liquid-solid contact time, as shown in Figure 6b, which also demonstrates that the process of de-pinning dominates the contact time. Furthermore, the moving ASHS can not only reduce the contact time at almost all impacting velocities, but can also produce a more rapid de-pinning behavior as compared to that of the stationary ASHS. Repeated wetting can also be easily avoided because the points of the initial interaction and lift-off do not coincide.

(a)

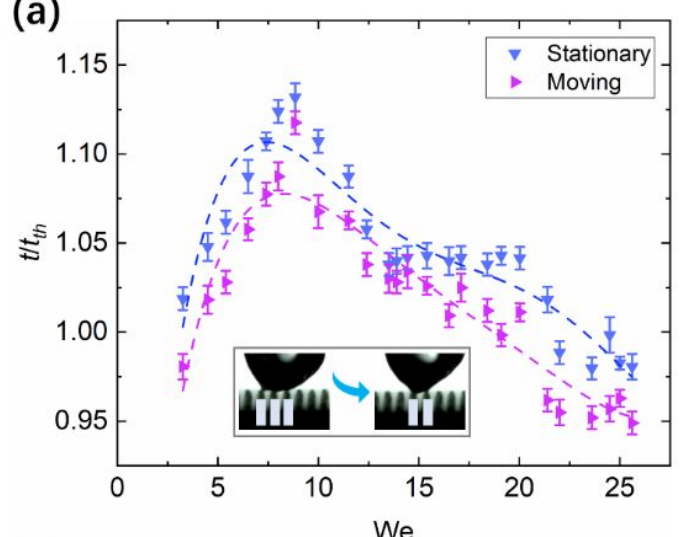

(c)

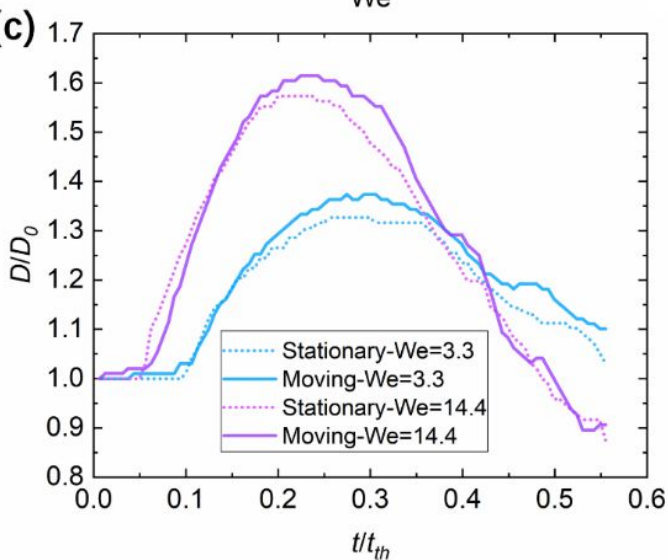

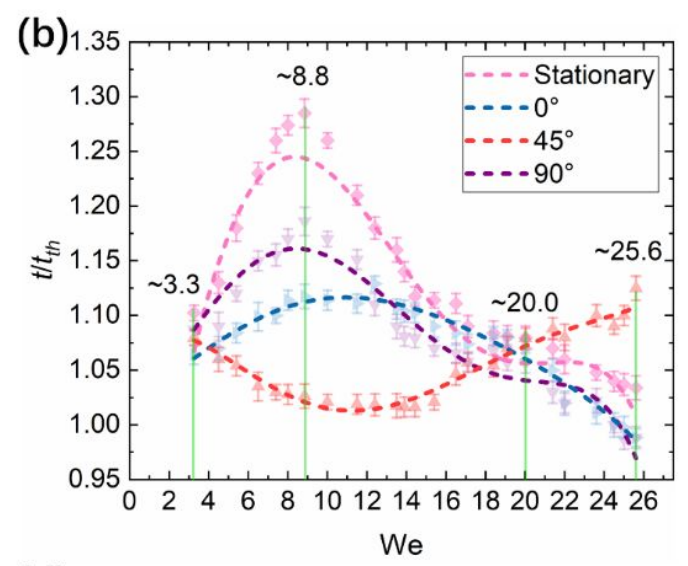

(d)

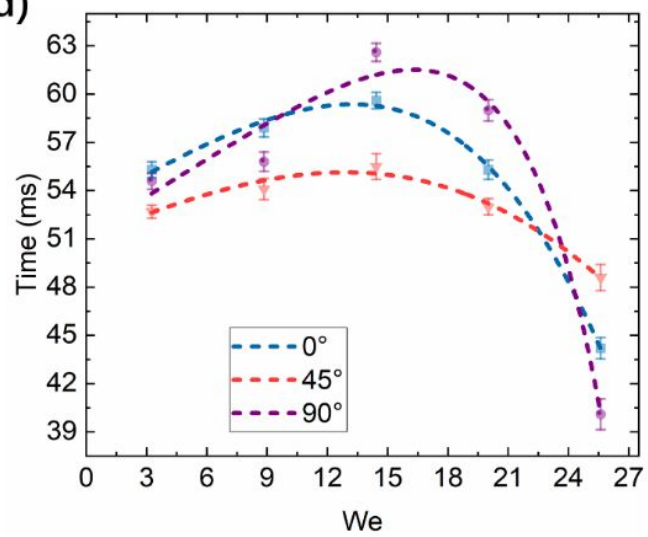

Figure 6. Comparisons of the time-resolved evolution of the droplet diameter and time-scale comparisons of the selected typical de-pinning process. (a) Comparison of the time-resolved evolution ( $t$ is normalized by $t_{t h}$ ) as a function of $W e$ on stationary and moving substrates. The time in which the number of selected typical interactive ribbings (marked in gray) change from three to two is investigated, and it is revealed that the moving ASHS reduces the pinning time. (b) Comparative analysis of the solid-liquid contact time ( $t$ is normalized by $t_{t h}$ ). The green line divides the fitted dotted curve into low $(3.3 \leq W e<8.8)$, medium $(8.8 \leq W e<20.0)$, and high $(20.0 \leq W e<25.6)$ Weber number regimes. The moving ASHS is shown to reduce the contact time. (c) Comparison of the diameter of a 
droplet (normalized by $D_{0}$ ) impacting the stationary and moving ASHSs ( $W e=3.3$ and $W e=14.4$ ) as a function of time-resolved evolution (normalized by $t_{t h}$ ). The droplets can spread more quickly to their maximum size when impinging on the moving ASHS under the higher Weber number regime (see the purple solid line). In addition, a larger liquid-solid contiguous area is observed on the moving ASHS, in which the dotted line is almost below the solid line (see Supporting Movies 2, 3, and 5). The error bars are based on three repeated experiments; (d) The hang time of the droplet as a function of We. The fitted dotted line indicates that an included angle of $\sim 45^{\circ}$ can significantly reduce the hang time in the Weber number range (see Supporting Movie 6).

\subsection{The tumbling droplet: a unique, active, and efficient dewatering strategy}

Despite previous insight into the directed rebounding of droplets being attributed to an inclined substrate ${ }^{27}$ or microstructures with extraordinary microscale gradients ${ }^{44}$, little is known about how directed rebounding is affected by a flat substrate with a uniform gradient. Note that the directed rebounding of droplets was observed on the selected moving ASHSs (the included angles were $0^{\circ}$, $45^{\circ}$, and $90^{\circ}$ ) containing horizontal and uniform ribbings via chronophotography ${ }^{45}$, as shown in Figure 7a $(W e=14.4$, see also Supporting Movie 6). All droplets were emitted at an oblique angle and tumbled downstream instead of repeatedly bouncing and falling vertically ${ }^{13}$. The hang times of the droplets from the initial contact with the substrate to their first landing downstream were quantified for the three included angles (Figure 6d). It is clear that the bouncing mode of droplets is dominated by the angle, and the droplet at a $45^{\circ}$ angle was confirmed to land first in the downstream substrate below the medium Weber numbers $(W e=3.3-20.0)$. A more noticeable oscillation occurred in the parabolic trajectory as the angle increased. This phenomenon is attributed to the increasing unbalanced de-pinning velocities, which are positively correlated with the included angle as the droplet is about to bounce off. Qualitative simulation (Figure 7a insets, $W e=17.8$ ) confirms this inference, and strong consistency is exhibited in Figure 3d.

The unbalanced de-pinning mechanism that creates the parabolic effect was also evaluated via simulation. The purple $(6.54 \mathrm{~ms})$ and blue $(6.66 \mathrm{~ms})$ rectangles in Figure $7 \mathbf{b}$ respectively correspond to the typical de-pinning step in Figure 5d, as the droplet is able to rebound backward (see Supporting Movie 4). Initially, the liquid in the groove is ejected vertically (the upward velocity vector, $V_{D e v}$ ) and is then replaced by air (purple rectangle, $t=6.54 \mathrm{~ms}$ ). As the substrate moves, the liquid closest to the solid edge is given the rightward velocity vector, while the adjacent upper liquid remains toward the center due to surface tension and viscous forces during the retraction process (blue rectangle, $t=6.66$ $\mathrm{ms}$ ). Eventually, the upward and inward velocity vectors are synthesized (the de-pinning velocity 
vector, $\left.V_{D e}\right)$, causing directed rebounding. In addition, the synthesized vectors accelerate the tumbling of the droplet with the synergistic effect of anisotropic superhydrophobicity and translational motion. It is therefore reasonable to assume that the criteria used to characterize the rapidly-shedding water is not solely the contact time of the solid-liquid interface ${ }^{12}$, but also the directed rebounding of droplets from essential aspects. Similar conditions that contribute to dehydration also occur when raindrops hit birds during flight. As predicted in this paper, the moving ASHS can actively drive the droplets to maintain directional rebounding for excellent droplet shedding, which should occur on an inclined substrate. Furthermore, even a flat moving ASHS can reduce the hang time by up to $23 \%$ as compared to a stationary superhydrophobic surface under a tilt angle of $\sim 5^{\circ}$, as confirmed by Hao et al. ${ }^{46}$.
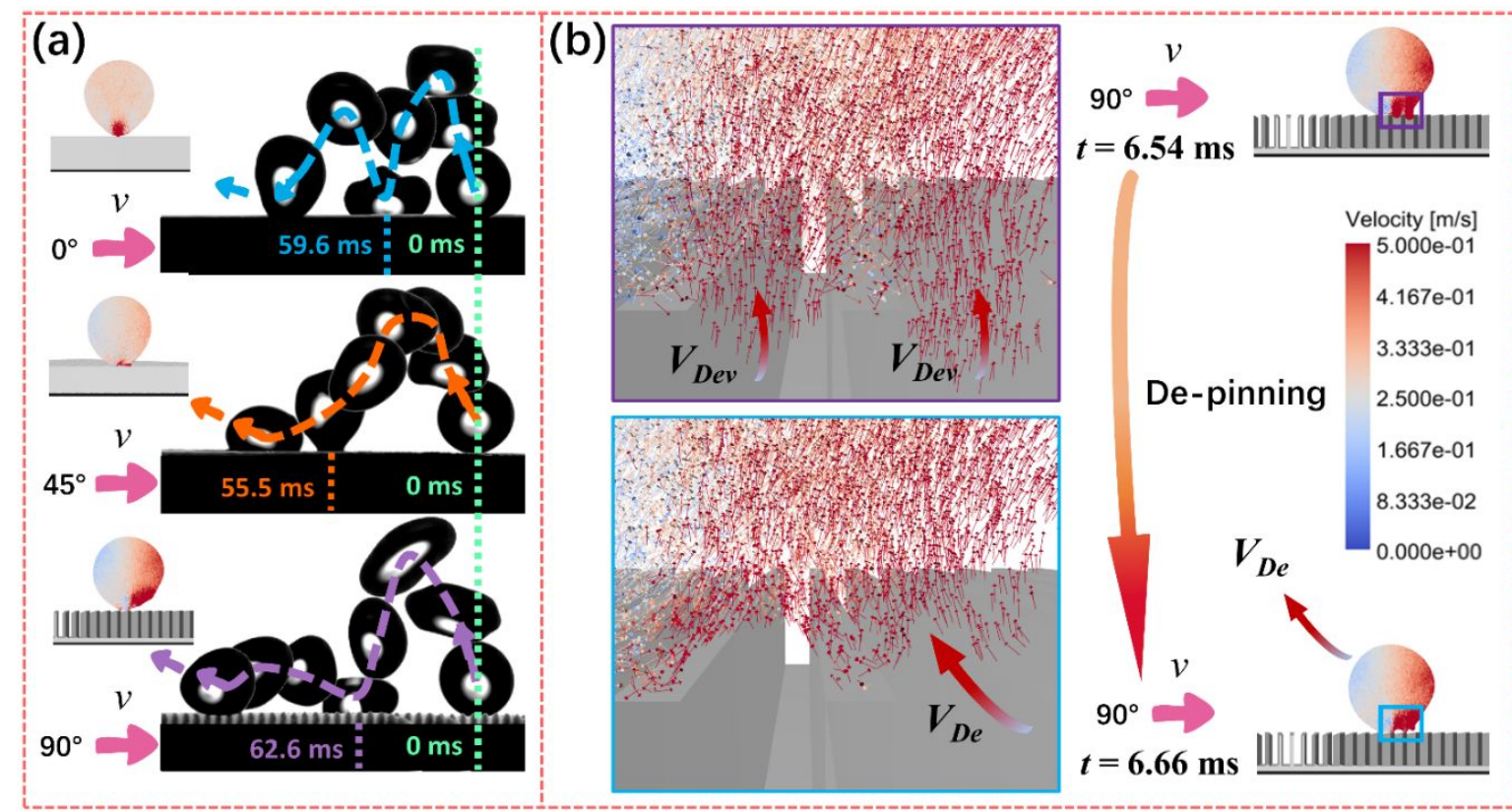

Figure 7. (a) Chronophotography of droplets impacting the moving ASHS with the included angles $0^{\circ}, 45^{\circ}$, and $90^{\circ}$, respectively $(W e=14.4)$. The superposition of successive frames reveals the parabolic paths of the directed bouncing droplets. The green dotted line represents the initial moment of collision, and the other corresponding colored dotted lines are the first landing moments. The insets illustrate that the simulations are qualitatively similar to the experimental parabolic trajectory. As the unbalanced velocity field is enhanced, the droplets tend to release and oscillate. (b) Mechanism of the de-pinning of directed rebounding droplets. The liquid in the groove with upward vertical velocities $V_{D e v}$ creates ascending movement (purple rectangle at $t=6.54 \mathrm{~ms}$ ). When the droplet is about to accomplish de-pinning, the liquid adjacent to the substrate is driven to the right, while the upper portion remains in centripetal motion, resulting in a composite velocity consisting of upward and inward vectors ( $V_{\text {Dev }}$, blue rectangle at $t=6.66 \mathrm{~ms}$, see Supporting Movie 6).

The understanding of the hydrophobic effects of moving interfaces is critical for applications in daily life, aerospace and defense industry; however, the mechanistic assessment of this new strategy 


\section{ASSOCIATED CONTENT}

A liquid jet impacting a moving kingfisher in a laboratory environment (Supporting Movie S1) (AVI) Side and overhead views of a droplet $\left(\mathrm{D}_{0}=2.06 \mathrm{~mm}, W e=3.3\right)$ impacting the stationary ASHS (Supporting Movie S2) (AVI)

Side and overhead views of a droplet $\left(\mathrm{D}_{0}=2.06 \mathrm{~mm}, \mathrm{We}=14.4\right)$ impacting the stationary ASHS (Supporting Movie S3) (AVI)

Overhead view of droplets impacting the moving substrates with directional anisotropy (Supporting Movie S4) (AVI)

Side views of droplets impacting the moving ASHS with the included angles $0^{\circ}, 45^{\circ}$, and $90^{\circ}$, respectively (Supporting Movie S5) (AVI)

Side view of droplets impacting on the moving ASHS with the included angles, $0^{\circ}, 45^{\circ}$, and $90^{\circ}$, respectively ( $W e=14.4)$ (Supporting Movie S6) (AVI)

Images of the selected kingfisher feathers (Figure S1); SEM frames of the selected kingfisher feathers (Figure S2). Wettability characterization of a kingfisher's wing feathers (Figure S3). SEM frames of the ASHS (Figure S4). Snapshots of a droplet impacting a stationary target ASHS (Figure S5) (PDF) 


\section{AUTHOR INFORMATION}

\section{Corresponding Author}

Chengchun Zhang - Key Laboratory of Bionic Engineering (Ministry of Education), Jilin University, Changchun 130022, China; orcid.org/0000-0002-0930-1139; E-mail: jluzcc@jlu.edu.cn

\section{Authors}

Yihua Zheng - Key Laboratory of Bionic Engineering (Ministry of Education), Jilin University, Changchun 130022, China; orcid.org/0000-0003-3625-6959

Jing Wang - College of Physics, Jilin University, Changchun 130012, China

Liang Yang - Centre for Renewable Energy Systems, Cranfield University, Cranfield MK43 0AL, United Kingdom

Chun Shen - State Key Laboratory of Automotive Simulation and Control, Jilin University, Changchun, 130022, China

Zhiwu Han - Key Laboratory of Bionic Engineering (Ministry of Education), Jilin University, Changchun 130022, China

Liu Yan - Key Laboratory of Bionic Engineering (Ministry of Education), Jilin University, Changchun 130022, China

\section{Author contributions}

C.Z. and Y.Z. conceived the study. Y.L., Y.Z., and J.W. conducted the experiments. C.Z., Z.H., and J.Y., L.Y., and Y.Z. performed the simulations. Y.Z. wrote the paper. All authors analyzed the data, proofread the paper, and made comments.

\section{Notes}

The authors declare no competing financial interest

\section{ACKNOWLEDGMENTS}

This study was supported by the National Key Research and Development Program of China (Grant No. 2018YFA0703300 and 2016YFE0132900), the National Natural Science Foundation of China (Grant No. 51575227, 51875243, and 51706084), the Science and Technology Development Program of Jilin Province (Grant No. 20180101319JC), and the Ph.D. Interdisciplinary Research Funding Scheme of Jilin University (Grant No. 1018320182).

\section{REFERENCES}

(1) Cao, Y.; Wu, Z.; Xu, Z. Effects of Rainfall on Aircraft Aerodynamics. Prog. Aerosp. Sci. 2014, 71, 85-127. https://doi.org/10.1016/j.paerosci.2014.07.003.

(2) Voigt, C. C.; Schneeberger, K.; Voigt-Heucke, S. L.; Lewanzik, D. Rain Increases the Energy 
Cost of Bat Flight. Biol. Lett. 2011, 7 (5), 793-795. https://doi.org/10.1098/rsbl.2011.0313.

(3) Ortega-Jiménez, V. M.; Álvarez-Borrego, S.; Arriaga-Ramírez, S.; Renner, M.; Bridge, E. S. Takeoff Flight Performance and Plumage Wettability in Cassin's Auklet Ptychoramphus Aleuticus, Xantus's Murrelet Synthliboramphus Hypoleucus and Leach's Storm-Petrel Oceanodroma Leucorhoa. J. Ornithol. 2010, 151 (1), 169-177. https://doi.org/10.1007/s10336-009-0441-z.

(4) Withers, P. C. Aerodynamics and Hydrodynamics of the 'Hovering'Flight of Wilson's Storm Petrel. J. Exp. Biol. 1979, 80 (1), 83-91.

(5) Ortega-Jimenez, V. M.; Dudley, R. Flying in the Rain: Hovering Performance of Anna's Hummingbirds under Varied Precipitation. Proc. R. Soc. B Biol. Sci. 2012, 279 (1744), 39964002. https://doi.org/10.1098/rspb.2012.1285.

(6) Zhang, C.; Wu, Z.; Zhang, X.; Yue, Y.; Wang, J. Effect of Feather Elasticity of Kingfisher Wing on Droplet Impact Dynamics. J. Bionic Eng. 2018, 15 (4), 731-740. https://doi.org/10.1007/s42235-018-0061-5.

(7) Jiang, L.; Zhao, Y.; Zhai, J. A Lotus-Leaf-like Superhydrophobic Surface: A Porous Microsphere/Nanofiber Composite Film Prepared by Electrohydrodynamics. Angew Chem Int Ed Engl 2004, 43 (33), 4338-4341.

(8) Bormashenko, E.; Bormashenko, Y.; Stein, T.; Whyman, G.; Bormashenko, E. Why Do Pigeon Feathers Repel Water? Hydrophobicity of Pennae, Cassie-Baxter Wetting Hypothesis and Cassie-Wenzel Capillarity-Induced Wetting Transition. J. Colloid Interface Sci. 2007, 311 (1), 212-216. https://doi.org/10.1016/j.jcis.2007.02.049.

(9) Marmur, A.; Della Volpe, C.; Siboni, S.; Amirfazli, A.; Drelich, J. W. Contact Angles and Wettability: Towards Common and Accurate Terminology. Surf. Innov. 2017, 5 (1), 3-8. https://doi.org/10.1680/jsuin.17.00002.

(10) Wu, H.; Zhang, R.; Sun, Y.; Lin, D.; Sun, Z.; Pan, W.; Downs, P. Biomimetic Nanofiber Patterns with Controlled Wettability. Soft Matter 2008. https://doi.org/10.1039/b805570j.

(11) Zhang, C.; Zheng, Y.; Wu, Z.; Wang, J.; Shen, C.; Liu, Y.; Ren, L. Non-Wet Kingfisher Flying in the Rain: The Water-Repellent Mechanism of Elastic Feathers. J. Colloid Interface Sci. 2019, 541, 56-64. https://doi.org/10.1016/j.jcis.2019.01.070.

(12) Bird, J. C.; Dhiman, R.; Kwon, H. M.; Varanasi, K. K. Reducing the Contact Time of a Bouncing Drop. Nature 2013, 503 (7476), 385-388. https://doi.org/10.1038/nature12740.

(13) Yarin, A. L. L. DROP IMPACT DYNAMICS: Splashing, Spreading, Receding, Bouncing.... Annu. Rev. Fluid Mech. 2006, 38 (1), 159-192. https://doi.org/10.1146/annurev.fluid.38.050304.092144. 
(14) Richard, D.; Clanet, C.; Quéré, D. Surface Phenomena-Contact Time of a Bouncing Drop. Nature 2002, 1334 (1994), 0-1.

(15) Toosi, S. F.; Moradi, S.; Ebrahimi, M.; Hatzikiriakos, S. G. Microfabrication of Polymeric Surfaces with Extreme Wettability Using Hot Embossing. Appl. Surf. Sci. 2016. https://doi.org/10.1016/j.apsusc.2016.03.116.

(16) Toosi, S. F.; Moradi, S.; Kamal, S.; Hatzikiriakos, S. G. Superhydrophobic Laser Ablated PTFE Substrates. Appl. Surf. Sci. 2015. https://doi.org/10.1016/j.apsusc.2015.05.026.

(17) Raczkowska, J.; Stetsyshyn, Y.; Awsiuk, K.; Lekka, M.; Marzec, M.; Harhay, K.; Ohar, H.; Ostapiv, D.; Sharan, M.; Yaremchuk, I.; et al. Temperature-Responsive Grafted Polymer Brushes Obtained from Renewable Sources with Potential Application as Substrates for Tissue Engineering. Appl. Surf. Sci. 2017. https://doi.org/10.1016/j.apsusc.2017.03.001.

(18) Stetsyshyn, Y.; Raczkowska, J.; Budkowski, A.; Awsiuk, K.; Kostruba, A.; Nastyshyn, S.; Harhay, K.; Lychkovskyy, E.; Ohar, H.; Nastishin, Y. Cholesterol-Based Grafted Polymer Brushes as Alignment Coating with Temperature-Tuned Anchoring for Nematic Liquid Crystals. Langmuir 2016. https://doi.org/10.1021/acs.langmuir.6b02946.

(19) Xu, Z.; Zhao, Y.; Wang, H.; Zhou, H.; Qin, C.; Wang, X.; Lin, T. Fluorine-Free Superhydrophobic Coatings with PH-Induced Wettability Transition for Controllable OilWater Separation. ACS Appl. Mater. Interfaces 2016. https://doi.org/10.1021/acsami.5b11720.

(20) Fu, S.; Zhou, H.; Wang, H.; Niu, H.; Yang, W.; Shao, H.; Wang, J.; Lin, T. Superhydrophilic, Underwater Directional Oil-Transport Fabrics with a Novel Oil Trapping Function. ACS Appl. Mater. Interfaces 2019. https://doi.org/10.1021/acsami.9b06533.

(21) Fu, S.; Zhou, H.; Wang, H.; Ding, J.; Liu, S.; Zhao, Y.; Niu, H.; Rutledge, G. C.; Lin, T. Magnet-Responsive, Superhydrophobic Fabrics from Waterborne, Fluoride-Free Coatings. RSC Adv. 2018. https://doi.org/10.1039/c7ra10941e.

(22) Almohammadi, H.; Amirfazli, A. Understanding the Drop Impact on Moving Hydrophilic and Hydrophobic Surfaces. Soft Matter 2017, 13 (10), 2040-2053. https://doi.org/10.1039/C6SM02514E.

(23) Buksh, S.; Almohammadi, H.; Marengo, M.; Amirfazli, A. Spreading of Low-Viscous Liquids on a Stationary and a Moving Surface. Exp. Fluids 2019, 60 (4), 76. https://doi.org/10.1007/s00348-019-2715-4.

(24) Almohammadi, H.; Amirfazli, A. Asymmetric Spreading of a Drop upon Impact onto a Surface. Langmuir 2017, 33 (23), 5957-5964. https://doi.org/10.1021/acs.langmuir.7b00704.

(25) Shao, Y.; Zhao, J.; Fan, Y.; Wan, Z.; Lu, L.; Zhang, Z.; Ming, W.; Ren, L. Shape Memory Superhydrophobic Surface with Switchable Transition between "Lotus Effect" to "Rose Petal 
Effect." Chem. Eng. J. 2020. https://doi.org/10.1016/j.cej.2019.122989.

(26) Zhao, J.; Song, L.; Yin, J.; Ming, W. Anti-Bioadhesion on Hierarchically Structured, Superhydrophobic Surfaces. Chem. Commun. 2013. https://doi.org/10.1039/c3cc44971h.

(27) Liu, Y.; Moevius, L.; Xu, X.; Qian, T.; Yeomans, J. M.; Wang, Z. Pancake Bouncing on Superhydrophobic Surfaces. Nat. Phys. 2014, 10 (7), 515-519.

https://doi.org/10.1038/nphys2980.

(28) Moevius, L.; Liu, Y.; Wang, Z.; Yeomans, J. M. Pancake Bouncing: Simulations and Theory and Experimental Verification. Langmuir 2014, 30 (43), 13021-13032. https://doi.org/10.1021/la5033916.

(29) Pooley, C. M.; Furtado, K. Eliminating Spurious Velocities in the Free-Energy Lattice Boltzmann Method. Phys. Rev. E 2008, 77 (4), 046702. https://doi.org/10.1103/PhysRevE.77.046702.

(30) Zheng, Y.; Zhang, C.; Wang, J.; Liu, Y.; Shen, C.; Yang, J. Robust Adhesion of Droplets via Heterogeneous Dynamic Petal Effects. J. Colloid Interface Sci. 2019, 557, 737-745. https://doi.org/10.1016/j.jcis.2019.09.070.

(31) Li, Z.; Kong, Q.; Ma, X.; Zang, D.; Guan, X.; Ren, X. Dynamic Effects and Adhesion of Water Droplet Impacts on Hydrophobic Surfaces: Bouncing or Sticking. Nanoscale 2017, 9 (24), 8249-8255. https://doi.org/10.1039/C7NR02906C.

(32) Vaikuntanathan, V.; Sivakumar, D. Maximum Spreading of Liquid Drops Impacting on Groove-Textured Surfaces: Effect of Surface Texture. Langmuir 2016, 32 (10), 2399-2409. https://doi.org/10.1021/acs.langmuir.5b04639.

(33) Guo, C.; Zhao, D.; Sun, Y.; Wang, M.; Liu, Y. Droplet Impact on Anisotropic Superhydrophobic Surfaces. Langmuir 2018, 34 (11), 3533-3540. https://doi.org/10.1021/acs.langmuir.7b03752.

(34) Clavijo, C. E.; Crockett, J.; Maynes, D. Effects of Isotropic and Anisotropic Slip on Droplet Impingement on a Superhydrophobic Surface. Phys. Fluids 2015, 27 (12). https://doi.org/10.1063/1.4936899.

(35) Han, J.; Ryu, S.; Kim, H.; Sen, P.; Choi, D.; Nam, Y.; Lee, C. Anisotropic Drop Spreading on Superhydrophobic Grates during Drop Impact. Soft Matter 2018, 14 (19), 3760-3767. https://doi.org/10.1039/c8sm00259b.

(36) Withers, B. Y. P. C. Aerodynamics and Hydrodynamics of the "hovering" Flight of Wilson's Storm-Petrel. J. Exp. Biol. 1987, 80 (1), 83-91.

(37) Bartolo, D.; Josserand, C.; Bonn, D. Retraction Dynamics of Aqueous Drops upon Impact on Non-Wetting Surfaces. J. Fluid Mech. 2005. https://doi.org/10.1017/S0022112005007184. 
(38) Aurélie Lafuma, D. Q. Superhydrophobic States. Nat. Mater. 2003, 2 (7), 457-460. https://doi.org/10.1038/nmat924.

(39) Yamamoto, K.; Ogata, S. 3-D Thermodynamic Analysis of Superhydrophobic Surfaces. J. Colloid Interface Sci. 2008, 326 (2), 471-477. https://doi.org/10.1016/j.jcis.2008.06.044.

(40) Lee, T.; Charrault, E.; Neto, C. Interfacial Slip on Rough, Patterned and Soft Surfaces: A Review of Experiments and Simulations. Adv. Colloid Interface Sci. 2014, 210, 21-38. https://doi.org/10.1016/j.cis.2014.02.015.

(41) Belyaev, A. V.; Vinogradova, O. I. Effective Slip in Pressure-Driven Flow Past SuperHydrophobic Stripes. J. Fluid Mech. 2010, 652, 489-499. https://doi.org/10.1017/S0022112010000741.

(42) Liu, Y.; Andrew, M.; Li, J.; Yeomans, J. M.; Wang, Z. Symmetry Breaking in Drop Bouncing on Curved Surfaces. Nat. Commun. 2015, 6, 1-8. https://doi.org/10.1038/ncomms10034.

(43) Richard, D.; Clanet, C.; Quéré, D. Surface Phenomena: Contact Time of a Bouncing Drop. Nature 2002, 417 (6891), 811.

(44) Malouin, B. A.; Koratkar, N. A.; Hirsa, A. H.; Wang, Z. Directed Rebounding of Droplets by Microscale Surface Roughness Gradients. Appl. Phys. Lett. 2010, 96 (23), 94-97. https://doi.org/10.1063/1.3442500.

(45) Soto, D.; Girard, H. L.; Le Helloco, A.; Binder, T.; Quéré, D.; Varanasi, K. K. Droplet Fragmentation Using a Mesh. Phys. Rev. Fluids 2018, 3 (8), 1-10. https://doi.org/10.1103/PhysRevFluids.3.083602.

(46) Hao, C.; Li, J.; Liu, Y.; Zhou, X.; Liu, Y.; Liu, R.; Che, L.; Zhou, W.; Sun, D.; Li, L.; et al. Superhydrophobic-like Tunable Droplet Bouncing on Slippery Liquid Interfaces. Nat. Commun. 2015, 6, 1-7. https://doi.org/10.1038/ncomms8986. 
(a)

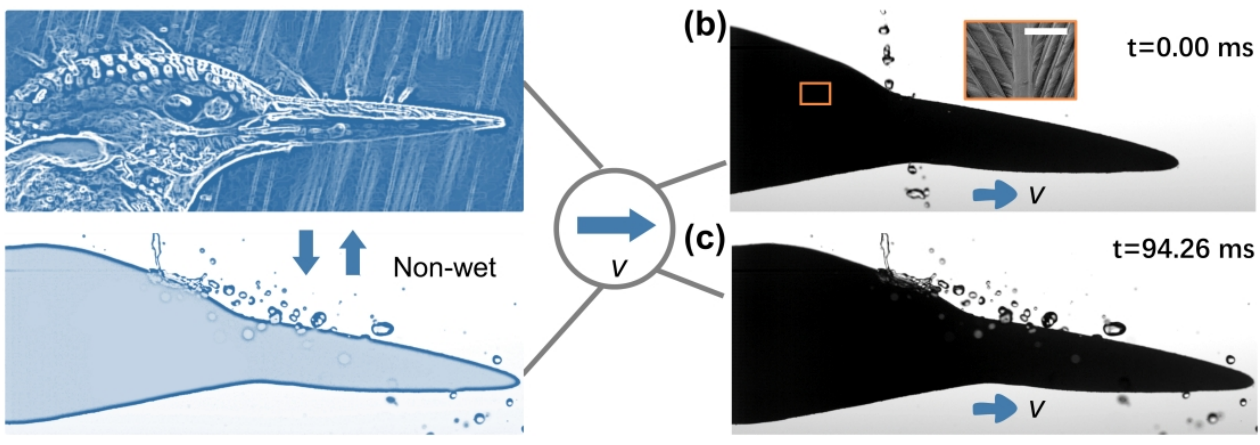

Remarkable water repellency of kingfishers in both natural and laboratory environments. 


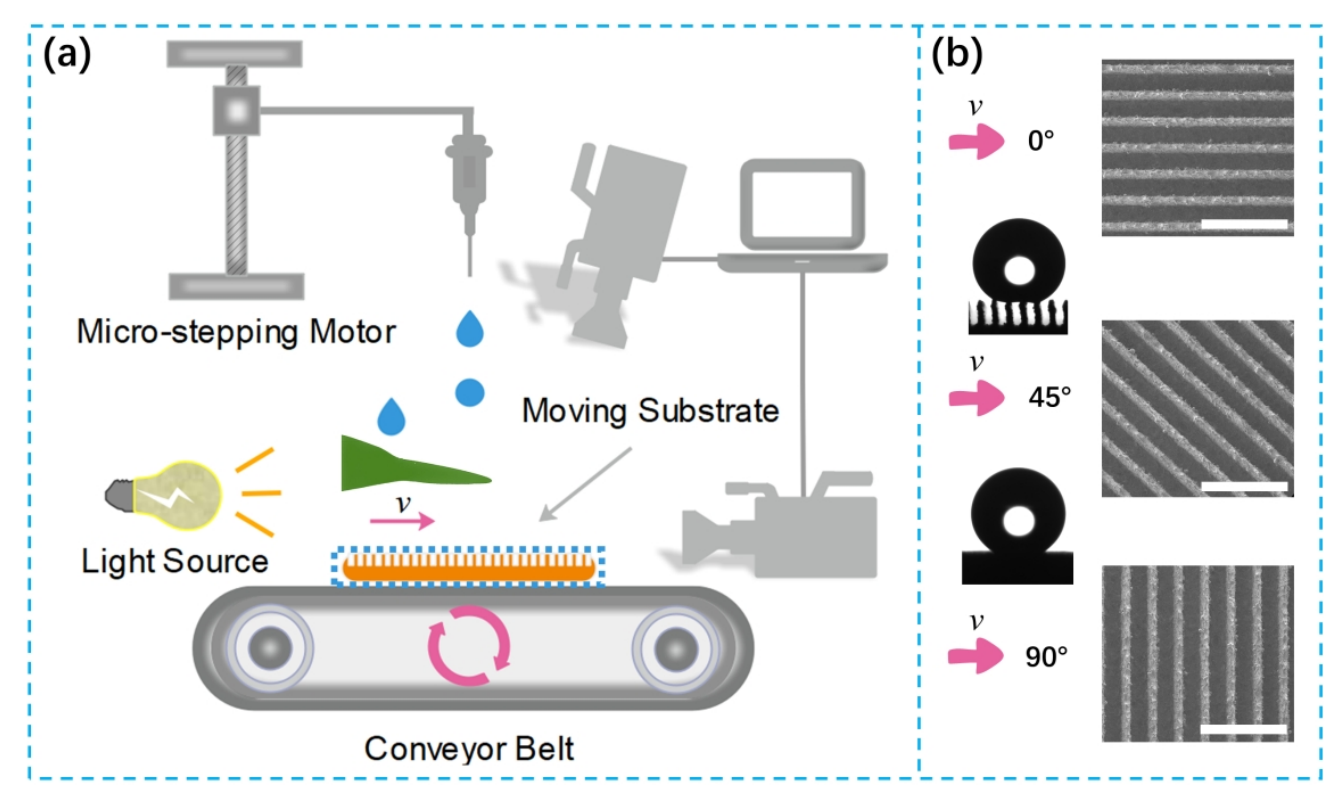

Schematic diagram of droplets impacting the three selected moving anisotropic substrates. 


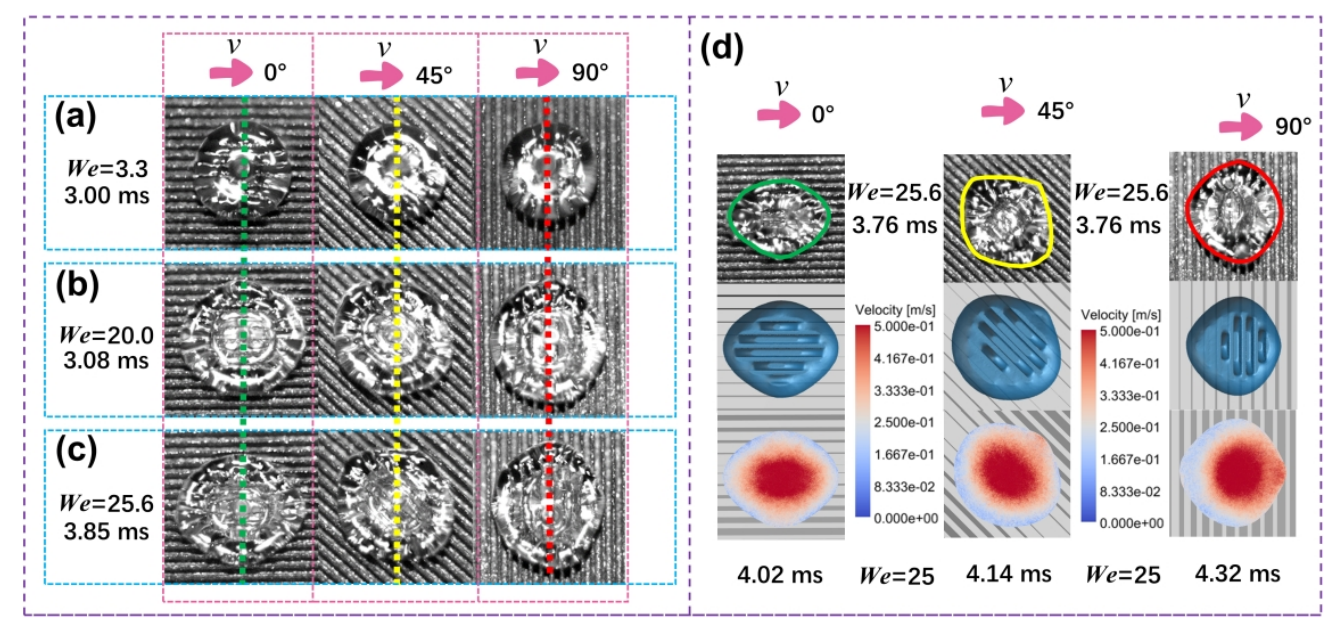

Overhead views of droplets impacting moving substrates with directional anisotropy. 

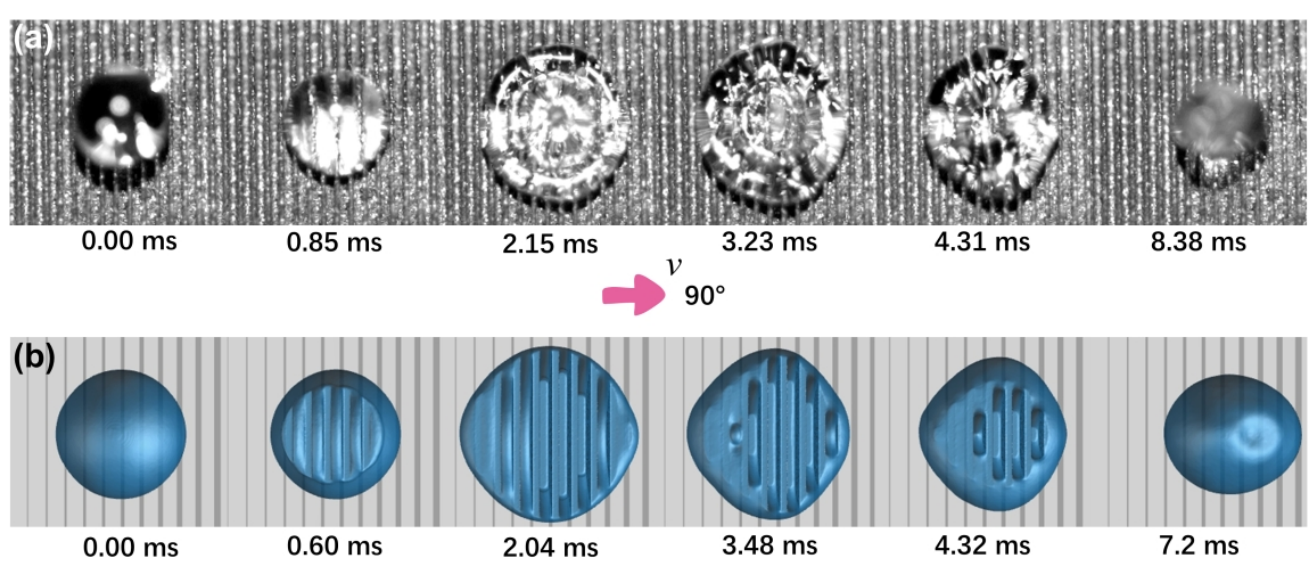

Snapshots of a droplet impacting a moving ASHS (We $=20,90^{\circ}$, vs $=0.13$ ) 
(a)

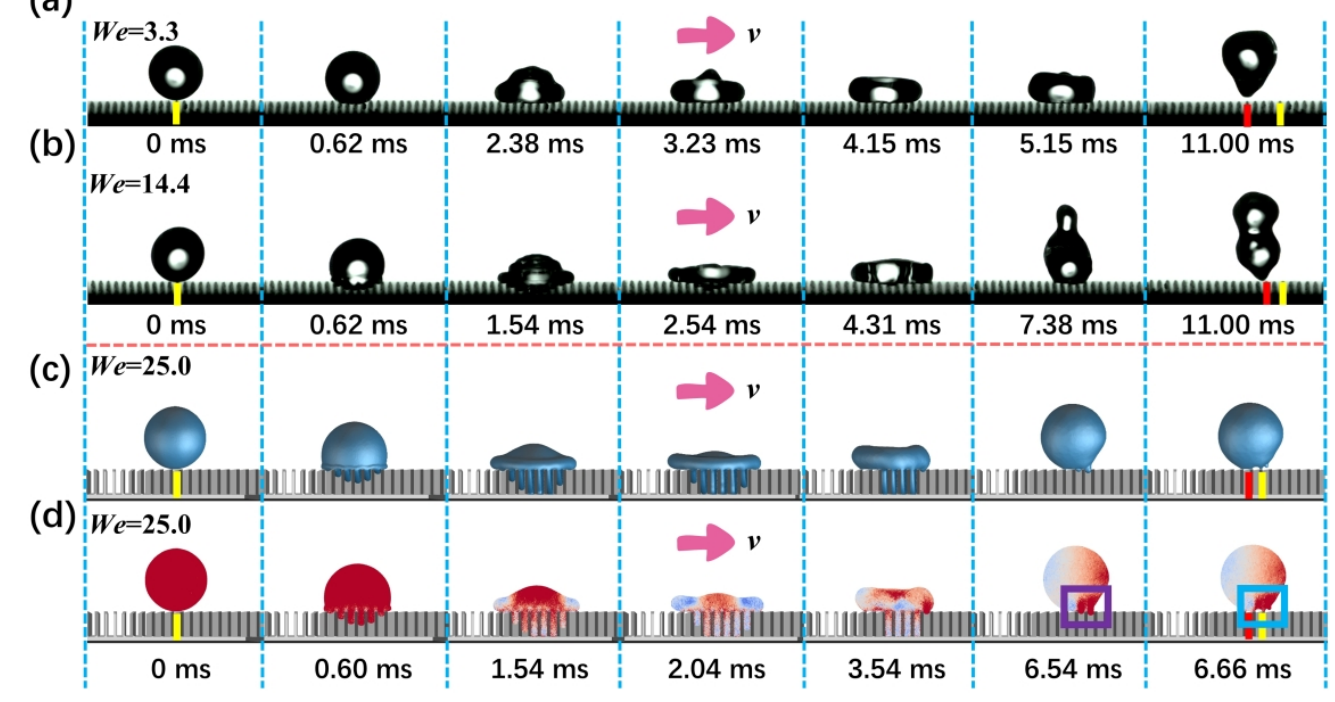

Sequential images of a droplet impacting the moving target ASHS $\left(90^{\circ}\right)$. 
(a)

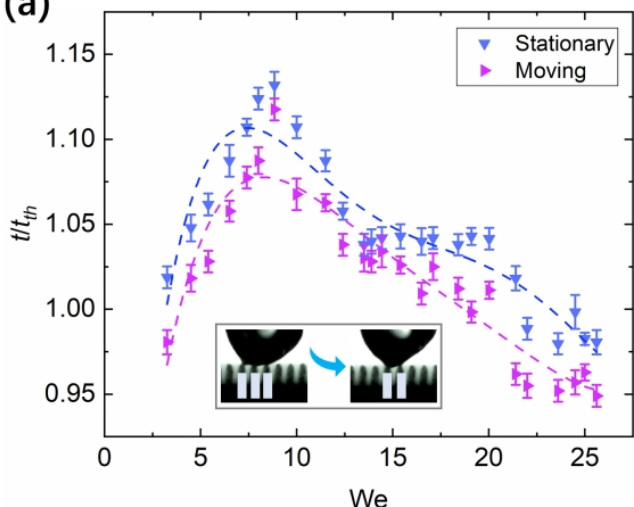

(c)

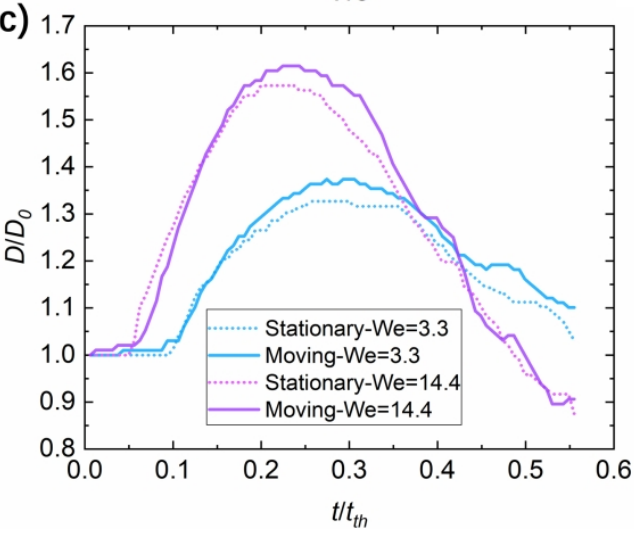

(b) 1

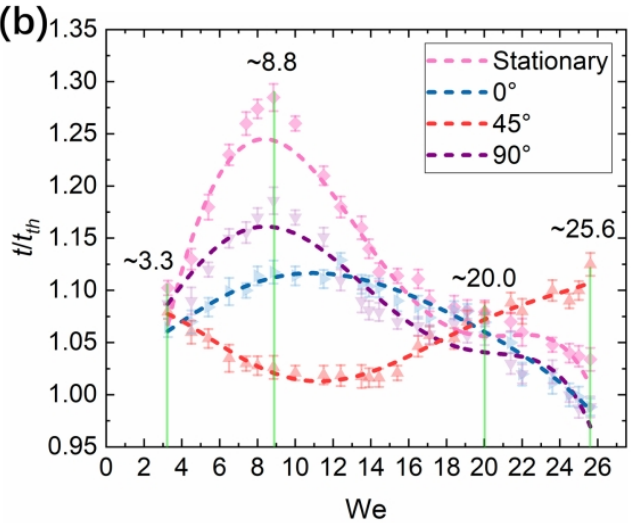

(d)

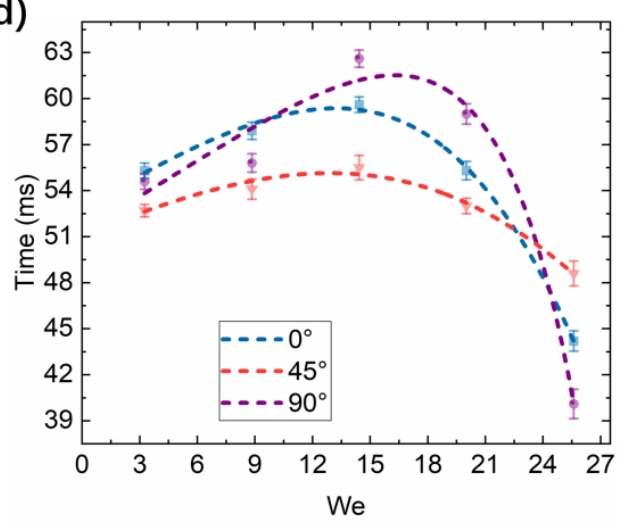

Comparisons of the time-resolved evolution of the droplet diameter and time-scale comparisons of the selected typical de-pinning process. 


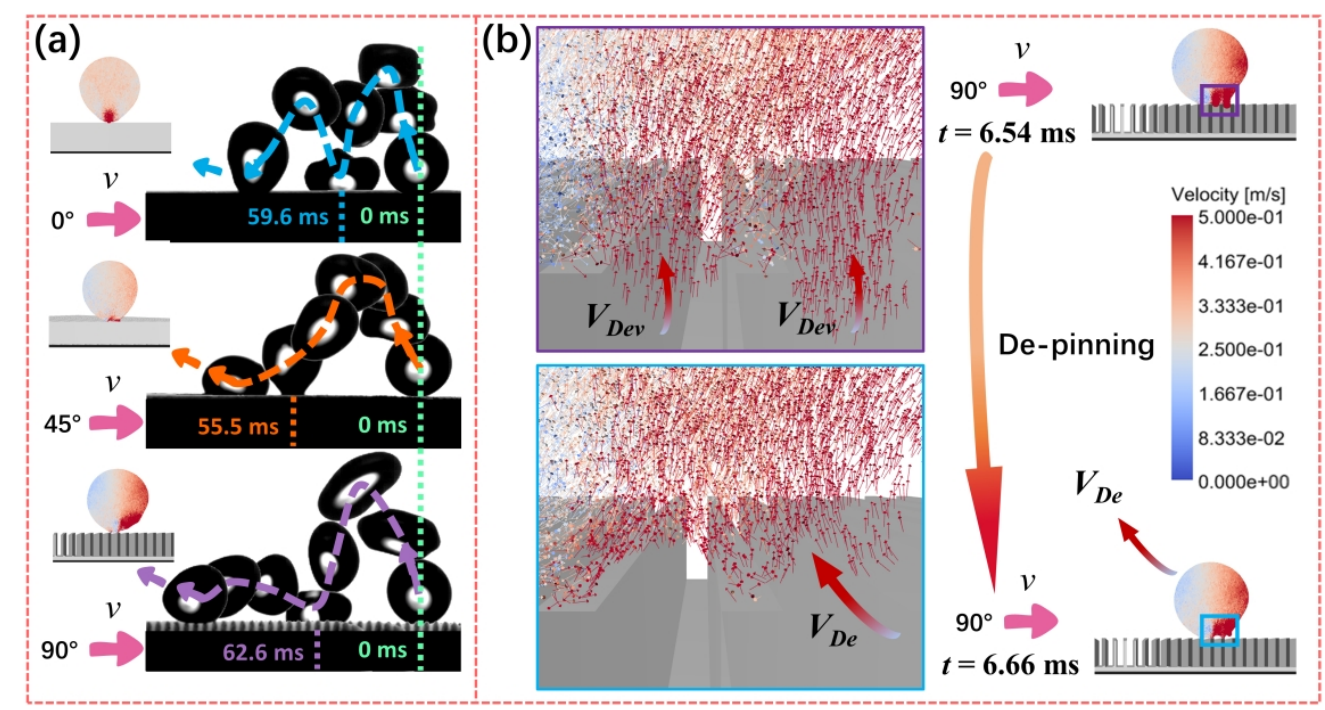

The tumbling droplet: a unique, active, and efficient dewatering strategy 